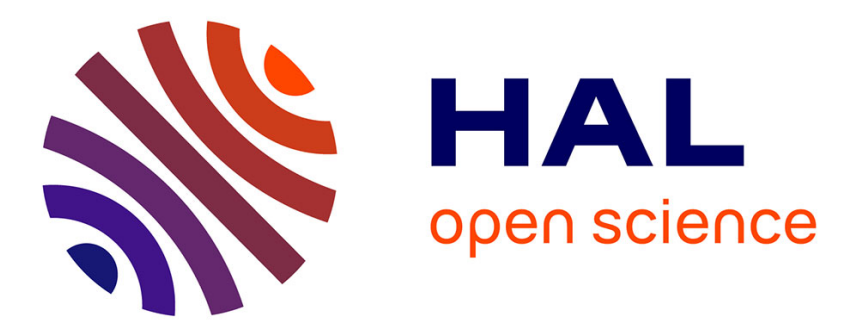

\title{
High electrically conductive composites of Polyamide 11 filled with silver nanowires: Nanocomposites processing, mechanical and electrical analysis
}

Antoine Lonjon, Ivan Caffrey, Delphine Carponcin, Eric Dantras, Colette

Lacabanne

\section{To cite this version:}

Antoine Lonjon, Ivan Caffrey, Delphine Carponcin, Eric Dantras, Colette Lacabanne. High electrically conductive composites of Polyamide 11 filled with silver nanowires: Nanocomposites processing, mechanical and electrical analysis. Journal of Non-Crystalline Solids, 2013, vol. 376, pp.199-204. 10.1016/j.jnoncrysol.2013.05.020 . hal-00843561

\section{HAL Id: hal-00843561 \\ https://hal.science/hal-00843561}

Submitted on 11 Jul 2013

HAL is a multi-disciplinary open access archive for the deposit and dissemination of scientific research documents, whether they are published or not. The documents may come from teaching and research institutions in France or abroad, or from public or private research centers.
L'archive ouverte pluridisciplinaire HAL, est destinée au dépôt et à la diffusion de documents scientifiques de niveau recherche, publiés ou non, émanant des établissements d'enseignement et de recherche français ou étrangers, des laboratoires publics ou privés. 


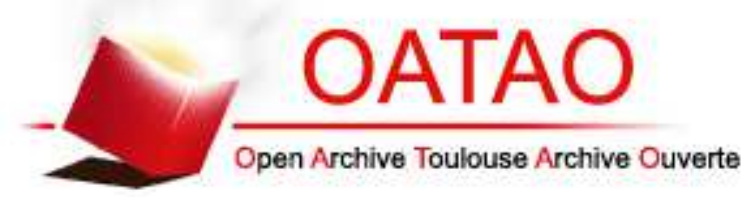

\section{Open Archive TOULOUSE Archive Ouverte (OATAO)}

OATAO is an open access repository that collects the work of Toulouse researchers and makes it freely available over the web where possible.

This is an author-deposited version published in : http://oatao.univ-toulouse.fr/ Eprints ID : 9200

To link to this article : $\underline{\text { http://dx.doi.org/10.1016/j.jnoncrysol.2013.05.020 }}$

\section{To cite this version :}

Lonjon, Antoine and Caffrey, Ivan and Carponcin, Delphine and Dantras, Eric and Lacabanne, Colette High electrically conductive composites of Polyamide 11 filled with silver nanowires: Nanocomposites processing, mechanical and electrical analysis. (2013) Journal of Non-Crystalline Solids, vol. 376 . pp. 199-204. ISSN 0022-3093

Any correspondance concerning this service should be sent to the repository administrator: staff-oatao@inp-toulouse.fr 


\title{
High electrically conductive composites of Polyamide 11 filled with silver nanowires: Nanocomposites processing, mechanical and electrical analysis
}

\author{
Antoine Lonjon ${ }^{\mathrm{a}, *}$, Ivan Caffrey ${ }^{\mathrm{b}}$, Delphine Carponcin ${ }^{\mathrm{a}}$, Eric Dantras ${ }^{\mathrm{a}}$, Colette Lacabanne ${ }^{\mathrm{a}}$ \\ a Physique des Polymères, Institut Carnot CIRIMAT, Université Paul Sabatier, 118 route de Narbonne, 31062 Toulouse cedex 9, France \\ ${ }^{b}$ Physics and Chemistry of Advanced Materials, Trinity College Dublin, Dublin 2, Ireland \\ * Corresponding author. Tel.: + 33561557617. \\ E-mail address: antoine.lonjon@univ-tlse3.fr (A. Lonjon).
}

A B S T R A C T

Highly conductive composites of silver nanowires and semicrystalline polyamide 11 for different content were prepared. A new method was developed to introduce nanowires: a combination of solvent way and an extrusion process. This technic provides pellets of composites directly suitable for sample molding. The silver nanowires poured in polyamide 11 were obtained by polyol process to reach large volume of nanowires. The dispersion of nanowires was evaluated by scanning electron microscopy and confirmed by a very low percolation threshold around 0.59 vol.\%. The level of conductivity above the percolation threshold is about $10^{2} \mathrm{~S} \mathrm{~m}^{-1}$. Differential scanning calorimetry and dynamic mechanical analyses have shown a stability of the composite physical structure and an optimization of the mechanical properties as a function of nanowires content until 3.6 vol.\%. A simultaneous enhancement of the electrical conductivity of polyamide 11 was obtained.

Keywords:

Nanowires;

Conductive polymer composites:

Polyamide 11;

Dynamic mechanical properties;

Nanocomposites

\section{Introduction}

A lot of works have been devoted to the elaboration of polymer conductive composites. Electrical conductivity is a crucial property for aeronautical composites polymer based. Conductive fillers are added to promote the increase of electrical conductivity. Metallic [1] or carbon micron particles [2,3] have been introduced and enable conductive composites at the expense of weight and mechanical properties. During the last decades new conductive particles have emerged such as carbon nanotube [4-6], carbon nanofibers [7], nanowires [8-10] with 1D geometry and submicrometer size. The interest of these new particles lies in their high aspect ratio. High values of aspect ratio enable the conductivity for lower rate of fillers. These low filled conductive polymer composites ( $<5$ vol.\%) generate a new class of multifunctional materials giving the conductivity of traditional conductive composite while preserving the mechanical properties [11] and limiting the overweight. To our knowledge, the best compromise to reach the higher electrical conductivity with the lower percolation threshold was obtained with metallic nanowires [10]. Silver nanowires elaborated by polyol process yields large volume of nanowires with high aspect ratio [12,13].

Polyamide 11 is a semi-crystalline thermoplastic with a low humidity permeability which allows a wide range of industrial applications like automotive, transportation and fluids industry. High electrically conductive composites of Polyamide 11 enable new conductive applications for these industries like protection of sensitive electrical systems.

The current method used for mixing particles with polyamide is the melt compounding. This technique requires a single or a twin screw extruder where the melt polymer and particles were poured. This technique gives pellets of composites. This technique provides generally interesting results for clays dispersion and for low aspect ratio particles [14]. Particles with high aspect ratio enhance largely the viscosity for very low rate of filler [15]. This phenomenon induces high shear stress during the mixing. It is hardly compatible with the preservation of the initial high aspect ratio of nanowires. The selective laser sintering is a different process currently used to elaborate three dimensional objects but rarely for composites [16]. The solvent dissolution of polyamide in the particles dispersion and precipitation reaches the best results for homogeneous dispersion. But the pellets obtained make it difficult to process in industrial conditions.

The aim of this study is to combine the advantages of processing methods. First, the solvent way was used to reach the lower percolation threshold with keeping the initial aspect ratio of nanowires. Then extrusion process was used to obtain composite pellets for sample elaboration. This study was particularly motivated by the 
desire to simultaneously enhance the electrical conductivity of polyamide 11 while preserving mechanical properties.

\section{Experimental section}

\subsection{Materials and sample preparation}

\subsubsection{Ag nanowires growth}

Electroless silver nanowires were synthesized by reducing $\mathrm{AgNO}_{3}$ with ethylene glycol in presence of poly(vinyl pyrrolidone)[13]. The reaction was carried out at $160{ }^{\circ} \mathrm{C}$ in a round-bottom balloon with magnetic stirring bar. This technique and the solution concentrations were described by Sun [12,13]. This technique allows us to obtain Ag nanowires (NWs) with a length distribution between 30 and $60 \mu \mathrm{m}$ and a diameter around 200 to $300 \mathrm{~nm}$. The Ag NWs morphology was characterized by scanning electron microscopy (SEM) to estimate the mean aspect ratio $(\xi=L / d)$. The nanowire suspension was rinsed in water and then filtered through a polyamide (200 nm pore size) membrane. Filtered nanowires were stored in acetone and dispersed using a short sonication pulse for $5 \mathrm{~s}$, corresponding to a dissipated power of $25 \mathrm{~W}$. A droplet of this solution was deposited onto a SEM pin.

\subsubsection{Nanocomposite processing}

Polyamide 11 (PA11) was supplied in granules by Arkema (France). The composites were prepared in two steps. In the first step, the mixing of nanowires and PA11 was prepared with using solvent casting method. PA11 was dissolved in a mixture of formic acid and dichloromethane (33.3-66.7 vol.\%). The nanowires dichloromethane (DCM) suspension was poured into the polymer solution and the mixture was sonicated to $5 \mathrm{~s}$ short pulse. Sonication parameters were optimized by the observation of Ag NWs dispersion using SEM [9]. The solvent was evaporated using a magnetic stirrer at $110{ }^{\circ} \mathrm{C}$ for $1 \mathrm{~h}$. Pellets of randomly dispersed Ag NWs in PA11 matrix were obtained and washed with distilled water to remove acid traces. In the second step, the pellets were melted at $200{ }^{\circ} \mathrm{C}$ and extruded with a die diameter of $1 \mathrm{~mm}$. The composites extruded were cut in pellets and pressed into a steel mold with a piston to form a sample at $200{ }^{\circ} \mathrm{C}$ under $0.3 \mathrm{MPa}$. Sample geometry for conductivity measurement is a disc with $20 \mathrm{~mm}$ in diameter and $1 \mathrm{~mm}$ in thickness. For mechanical study, the sample geometry is a $40 \mathrm{~mm}$ length, $10 \mathrm{~mm}$ width and $1 \mathrm{~mm}$ in thickness parallelepiped. No solvent residue was found by thermogravimetric analysis, indicating a complete removal of solvent from samples. Ag NWs/PA11 composites were elaborated with a volume fraction varying from 0 to 3.6 vol.\% with silver nanowires.

\subsection{Scanning electron microscopy}

The morphology of the silver nanowires was observed by scanning electron microscope using a JEOL JSM 6700F equipped with a field emission gun (SEM-FEG). Nanocomposite samples were cryocut at the liquid nitrogen temperature for observation by SEM.

\subsection{Differential scanning calorimetry}

Crystallization and melting phenomena were investigated using a TA Instruments 2920 differential scanning calorimeter (DSC). Before the heating and cooling runs, the samples were melted at $220{ }^{\circ} \mathrm{C}$ and maintained at this temperature for $5 \mathrm{~min}$ in order to erase the thermal history. The samples were then cooled from 200 to $45{ }^{\circ} \mathrm{C}$ at a rate of $20{ }^{\circ} \mathrm{C} \mathrm{min}{ }^{-1}$ and then maintained at $45{ }^{\circ} \mathrm{C}$ for $100 \mathrm{~min}$. The aim of this annealing was to highlight the glass transition temperature $\left(T_{\mathrm{g}}\right)$ with increasing the aging overshoot. Samples were then heated from $-10{ }^{\circ} \mathrm{C}$ to $220^{\circ} \mathrm{C}$ at a rate of $20{ }^{\circ} \mathrm{C} \mathrm{min}^{-1}$ and then cooled back to $-10{ }^{\circ} \mathrm{C}$ at the same rate. All the DSC measurements were performed under helium atmosphere. The glass transition was taken as the midpoint of the heat capacity step during the heating and the transition temperatures were taken as the peak maximum or minimum in the DSC thermograms. The crystallinity ratio of the PA11 composites was calculated according to the weight fraction of polymer using the calculated value of the theoretical $100 \%$ crystalline enthalpy value of PA11, $\Delta H_{0 \mathrm{~m}}=244 \mathrm{~J} \mathrm{~g}^{-1}$.

\subsection{Electrical conductivity}

To reduce contact resistivity with the cell electrodes, a thin gold layer $(100 \mathrm{~nm})$ was sputtered on the both samples sides using a BOC Edwards Scancoat Six sputter coater.

The electrical optimization of filled polymer composite is strongly dependent on the volume fraction $p$ and the aspect ratio $\xi$ of the filler. In three-dimensional disordered systems, the insulator to conductor transition is marked by the percolation threshold. This phenomena is driven by the percolation law [17].

$\sigma=\sigma_{0}\left(p-p_{c}\right)^{t}$

where $\sigma_{0}$ is a constant, $p_{\mathrm{c}}$ the percolation threshold and $t$ is the critical exponent dependent on the lattice dimension. Some models [18-20] described randomly dispersed composites with high aspect ratio particles like the nanowires. In the case of the percolation model for cylindrical sticks system, proposed by Balberg and Binenbaum [19] looks to be adequate. It shows that the percolation threshold is dependent from the aspect ratio and the anisotropy of sticks through their excluded volume $V_{\mathrm{ex}}$. At the percolation threshold, $V_{\mathrm{ex}}$ is found to be constant. When NWs are considered as randomly oriented sticks with $L / r>>1$, the critical excluded volume $V_{\mathrm{ex}}^{\mathrm{cr}}$ is between 1.4 and 1.8 and is given by the expression derivative from the Balberg's equation:

$V_{\mathrm{ex}}^{\mathrm{cr}}=\frac{L}{r} p_{\mathrm{c}}=1.6 \pm 0.2$

where $p_{\mathrm{c}}$ is the critical volume fraction for percolation.

\subsubsection{Low electrical conductivity values}

Bulk electrical conductivity of nanocomposites was carried out by two independent methods. For conductive composites filled below the percolation threshold the Novocontrol broadband spectrometer was used to record the complex conductivity $\sigma^{*}(\omega)$. The measurements were done in the frequency range from $10^{-2} \mathrm{~Hz}$ to $10^{6} \mathrm{~Hz}$ at room temperature. The real part $\sigma^{\prime}(\omega)$, of the complex conductivity $\sigma^{*}(\omega)$ was investigated. For all the nanocomposite samples considered in this study, the phase lag between the measured impedance and the applied ac voltage was negligible at low frequencies, so that the reported impedance at $0.01 \mathrm{~Hz}$ is equivalent to the dc resistance. The dc conductivity $\sigma_{\mathrm{dc}}$ of samples was determined from the independent frequency part of $\sigma^{\prime}(\omega)$ [4]; i.e. the low frequency plateau. Cylinders of $1 \mathrm{~mm}$ thickness were introduced between two circular gold plated electrodes $(20 \mathrm{~mm}$ in diameter).

\subsubsection{High electrical conductivity values}

The conductivity value reach above the percolation threshold was measured by four point-probe technique. The conductivity of nanocomposites cylinders was obtained with a Keithley 2420 SourceMeter in a four-point-probe configuration.

\subsection{Dynamic mechanical relaxation}

The mechanical behavior of composites was evaluated through the measurement of the shear modulus. The mechanic relaxation mode has been determined using the constant frequency-constant amplitude temperature scan method. The real part of the modulus, $G^{\prime}(T)$, and the imaginary part $G^{\prime \prime}(T)$ of the matrix and NWs filled polyamide composites were measured as function of temperature. Dynamic 

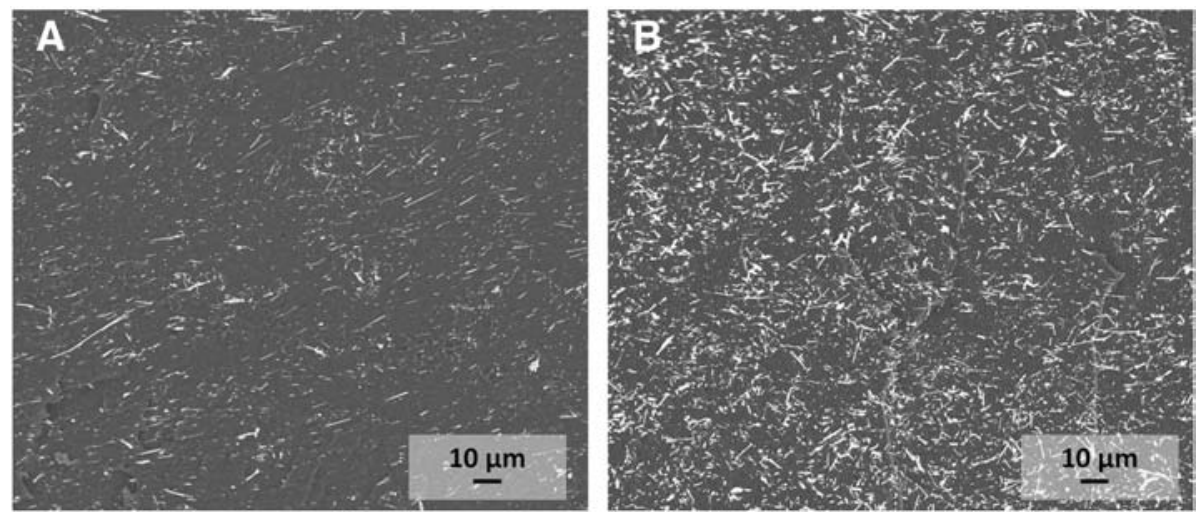

Fig. 1. SEM images of the fracture surface of silver nanowire/PA11 polymer composites filled at 1 vol.\% and 3.6 vol.\% at the same magnification.

mechanical analyses (DMA) were performed using an ARES (Advanced Rheometric Expansion System) strain controlled rheometer (TA Instruments). Tests were carried out in torsion rectangular mode over the linear elasticity range $\left(10^{-1} \%\right.$ strain at angular frequency of $\left.1 \mathrm{rad} \mathrm{s}^{-1}\right)$. This technique allows us to access to the complex shear modulus $G^{*}(\omega, T)$.

$G^{*}(\omega, T)=G^{\prime}(\omega, T)+i G^{\prime \prime}(\omega, T)$

\section{Results}

Ag NW/PA11 composites were prepared with a volume fraction of Ag NWs varying from 0 to 3.6 vol.\%. Figs. 1 and 2 show the SEM image of the polymer matrix filled by 1 and 3.6 vol.\% of Ag NWs for two magnifications. Silver nanowires are clearly individual and homogeneously dispersed in PA11 despite the presence of some little aggregates. The temperature and pressure conditions in cylinder applied for the compression molding nanocomposites sample processing avoid the slight orientation of the nanowires observed in form film samples [9]. Individual NWs are clearly observed for these rates. NWs have no affinity with DCM and keep their homogeneous dispersion in the polymer. Metallic nanowires contrary to CNTs do not develop electrostatic affinities and prevent them from agglomeration.

Influence of Ag NWs on the PA11 amorphous phase was studied on Fig. 3. A slight linear decrease of $T_{\mathrm{g}}$ was observed for composites. For the neat PA11, the $T_{\mathrm{g}}$ was measured at $58.1{ }^{\circ} \mathrm{C}$. The influence on crystalline phase was investigated with the observation of the crystallization peak. Fig. 4 shows the crystallization peak evolution and Fig. 5 shows the calculated crystallinity fraction for each composite; the maximum crystallization peak was observed for $162.4{ }^{\circ} \mathrm{C}$ and the crystallinity ratio of $16.1 \%$ was calculated. The crystallinity of the PA11 composites decreases slightly to $14.9 \%$ for the composite filled with 3.6 vol.\% of $\mathrm{Ag}$ NWs. The results obtained with Ni NWs in P(VDF-TrFE) have been added to be compared and discussed.

Table 1 and Fig. 6 show the electrical conductivity $\sigma_{\mathrm{dc}}$ obtained for $\mathrm{Ag}$ NWs/PA11 samples at room temperature as function of the $\mathrm{Ag}$ NWs volume fraction. The electrical conductivity of the insulating PA11 polymer is $10^{-12} \mathrm{~S} \mathrm{~m}^{-1}$. The conductivity fits the percolation law (Eq. (1)) for a percolation threshold value of 0.59 vol.\% of silver nanowires.

The real part of the modulus, $G^{\prime}(T)$, and the imaginary part $G^{\prime \prime}(T)$ were measured at $\omega=1 \mathrm{rad} \mathrm{s}^{-1}$ for samples filled with different $\mathrm{Ni}$ nanowires ratio. They are reported in Figs. 7 and 8 respectively. The temperature range was $-135{ }^{\circ} \mathrm{C}$ to $120{ }^{\circ} \mathrm{C}$ with a heating rate of $3{ }^{\circ} \mathrm{C} / \mathrm{min}$. The composites filled with NWs show an improvement of the conservative part of the shear modulus for low rate until $2.5 \%$ vol.. Fig. 8 focused on the $\alpha$ relaxation mode, the dynamical mechanical manifestation of the glass transition.

Fig. 9 shows the ratio of $G^{\prime}$ composite value to $G^{\prime}$ matrix one as function of the NWs content for the vitreous and the rubbery plateau. The vitreous and the rubbery plateau values have been chosen at $T_{\alpha}-50{ }^{\circ} \mathrm{C}$ and $T_{\alpha}+50{ }^{\circ} \mathrm{C}$ respectively. The results obtained with $\mathrm{Ni}$ NWs in P(VDF-TrFE) have been added to be compared and discussed.

\section{Discussion}

The PA11 amorphous phase is not modified by the introduction of silver NWs. The $T_{\mathrm{g}}$ evolution shown on Fig. 3 has a slight linear decrease of $1{ }^{\circ} \mathrm{C}$ for $3.63 \mathrm{vol} . \%$. This modification is not significant and this phenomenon has been associated with a strong intrinsic hydrogen bonds network generally observed in polyamides; these physical bonds network stabilize the PA11 amorphous phase. It is quite
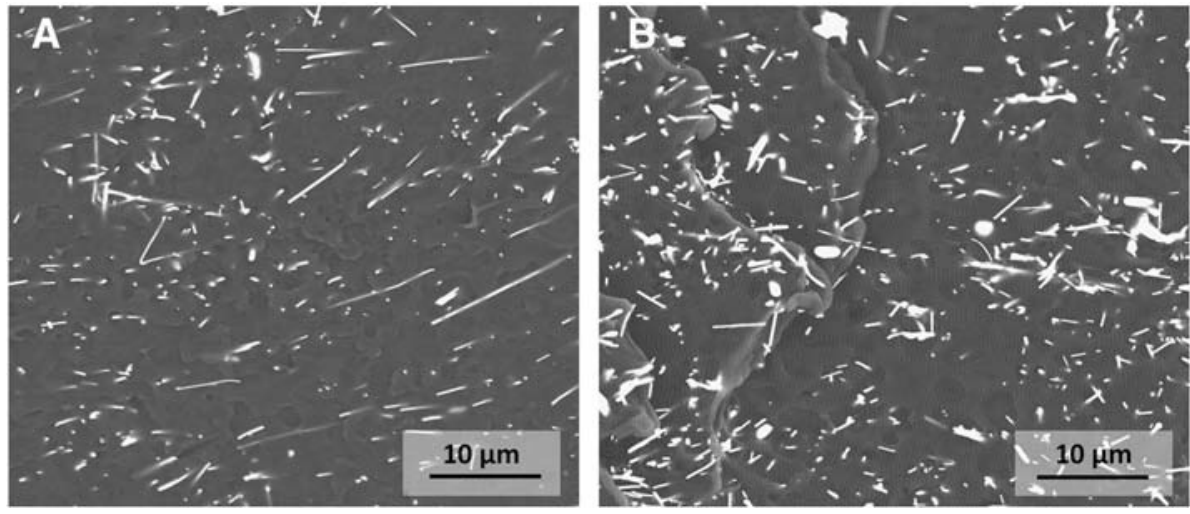

Fig. 2. SEM images of the fracture surface of silver nanowire/PA11 polymer composites filled at 1 vol.\% and 3.6 vol.\% at the same magnification. 

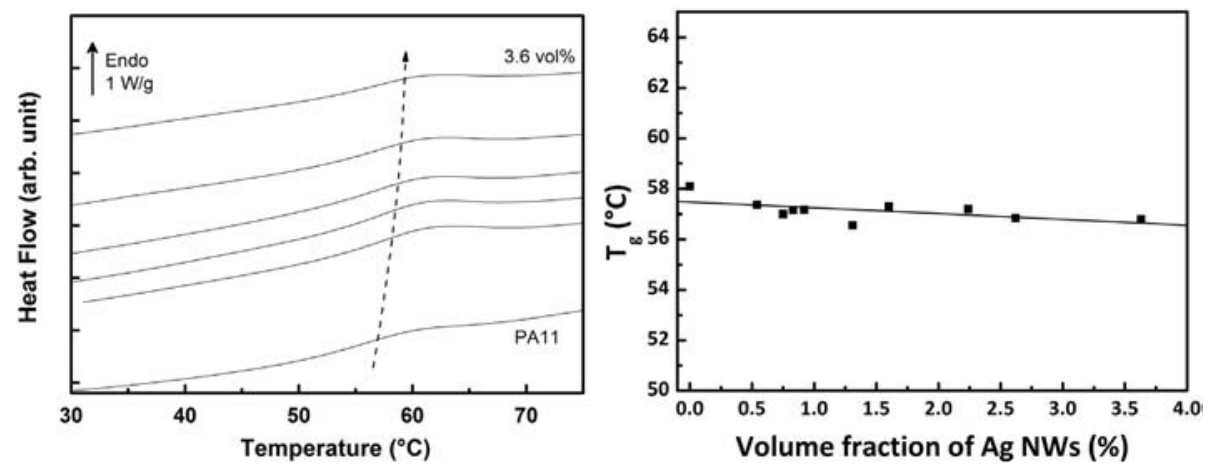

Fig. 3. Glass transition temperature $\left(T_{\mathrm{g}}\right)$ of PA11 matrix as a function of Ag nanowires volume fraction. Line corresponds to a guide line for the eyes.

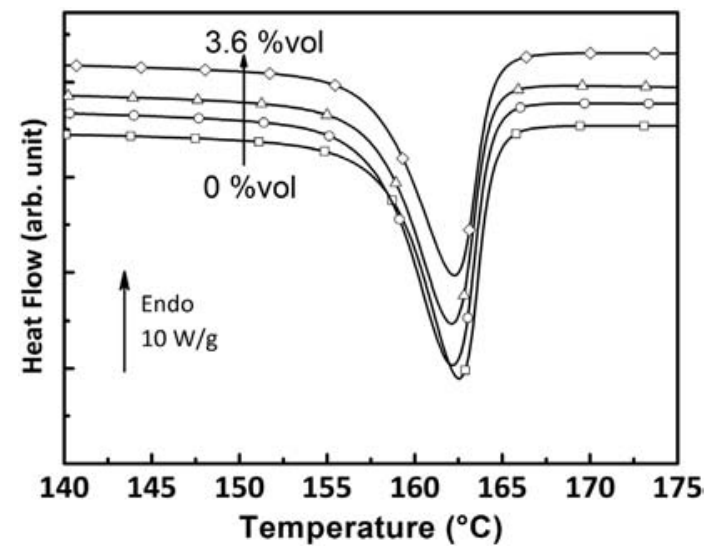

Fig. 4. Melting peak for neat PA 11 and its composites elaborated with $0,0.75,2.25$, and 3.6 vol.\% of NWs. Thermograms are recorded during the second temperature scan.

different from a previous result obtained with hydroxyapatite [21] or clays [14] nanoparticles.

The influence of NWs on PA11 physical structure was also followed by the crystallinity ratio. Fig. 4 shows the evolution of the crystallization peak with a slight decrease of $0.5{ }^{\circ} \mathrm{C}$ as soon as we poured Ag NWs for every volume fraction to 3.63 vol.\%. The peak width is constant reflecting the preservation of crystallites diameter. Ag NWs do not have any influence on crystallites morphology and no transcrystallinity phenomenon was observed. NWs do not play the role of nucleating agent, unlike nanoclays [22], CNTs [23] and nanohydroxyapatite [21]. The crystallinity ratio reported on Fig. 5 shows a low decrease around 6\% for 3.63 vol.\%. This value is lower

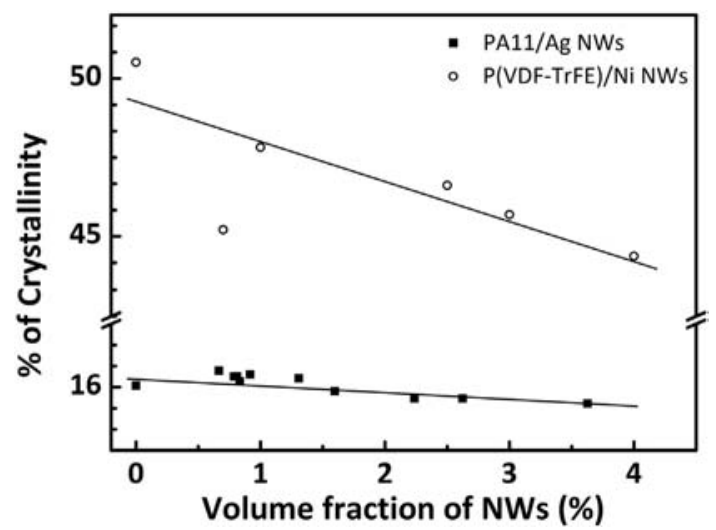

Fig. 5. Degree of crystallinity of PA11 matrix as a function of Ag nanowires volume fraction ( $\mathbf{\square})$ and $\mathrm{P}(\mathrm{VDF}-\mathrm{TrFE})$ matrix as a function of Ni nanowires volume fraction $(\mathrm{O})$ [24]. Lines correspond to a guide line for the eyes. than $\mathrm{P}(\mathrm{VDF}-\mathrm{TrFE}) /$ metallic nanowires composites [11,24]. This difference is explained by the strong physical network of amide groups which could be stronger than topological strain induced by nanowires.

The conductivity measurements of PA11/Ag NWs composites show an important step of conductivity associated with a percolation threshold behavior. The conductivity versus nanowires content follows the percolation law expressed in Eq. (1). The percolation threshold was measured near $0.59 \mathrm{vol} . \%$ of Ag NWs. This percolation threshold $p_{\mathrm{c}}$ is the lowest obtained for metallic nanowires dispersed in a polymer matrix $[8-10,24-26]$. The value of dimensionality factor t extrapolated from the Eq. ( 1 ) was equalled to $t=3$. It shows a divergence from the universal value of a 3D percolated system (1.94) randomly and homogeneously dispersed. This difference is explained by the presence of little aggregates [27]. Aggregates could slightly modify the dimensionality of the observed system [28]. This discrepancy was observed for some carbon nanotubes [29] or metal particles systems [30]. From the scaling law (Eq. (1)), we calculate the extrapolation at 100 vol.\% of conductive particles $\sigma_{(p=100 \%)}$ which corresponds to the electrical conductivity of Ag NWs bundles. The $\sigma_{(p=100 \%)}$ value was measured around $2 \times 10^{7} \mathrm{~S} \mathrm{~m}^{-1}$ and is in better agreement with the level of conductivity of silver bulk value $\left(6.5 \times 10^{7} \mathrm{~S} \mathrm{~m}^{-1}\right)$ than the dc conductivity of Ag NWs pressed in a sheet form $\left(1 \times 10^{5} \mathrm{~S} \mathrm{~m}^{-1}\right)$. The extrapolation value confirmed the presence of aggregates in composites. The apparent aspect ratio of Ag NWs could be extrapolated from the Eq. (2). The calculation gives an estimate aspect ratio value around 270 which fitting correctly the geometry of individual nanowires after elaboration. The level of conductivity around $100 \mathrm{~S} \mathrm{~m}^{-1}$ obtained above the percolation threshold is in a good agreement with other studies of metal particles dispersed in polymer matrix $[9,10,24,30-33]$. The conductivity value above $p_{c}$ is independent from the type of polymer matrix and particle geometry.

In Fig. 7, we note a classical stiffening behavior of the composites versus high aspect ratio content until 2.5 vol.\%. It confirmed previous results obtained for CNTs in PA11[34] or nickel NWs in P(VDF-TrFE) [11]. The $\alpha$ relaxation peak observed on Fig. 8 shows a weak decrease of the $T_{\alpha}$ temperature in the same range as observed on the $T_{\mathrm{g}}$ measured

Table 1

Nanocomposites: electrical conductivity versus volume fraction of Ag NWs.

\begin{tabular}{ll}
\hline \% vol. of Ag NWs & $\sigma_{\mathrm{dc}}\left(\mathrm{S} \mathrm{m}^{-1}\right)$ \\
\hline 0.67 & $2.4 \times 10^{-2}$ \\
0.75 & $5.7 \times 10^{-2}$ \\
0.78 & $2.4 \times 10^{-2}$ \\
0.83 & $7.4 \times 10^{-2}$ \\
0.92 & 2.7 \\
1.31 & 4.8 \\
1.59 & $3.7 \times 10^{1}$ \\
2.24 & $1.6 \times 10^{2}$ \\
2.62 & $2.4 \times 10^{2}$ \\
3.63 & $2.7 \times 10^{2}$ \\
\hline
\end{tabular}




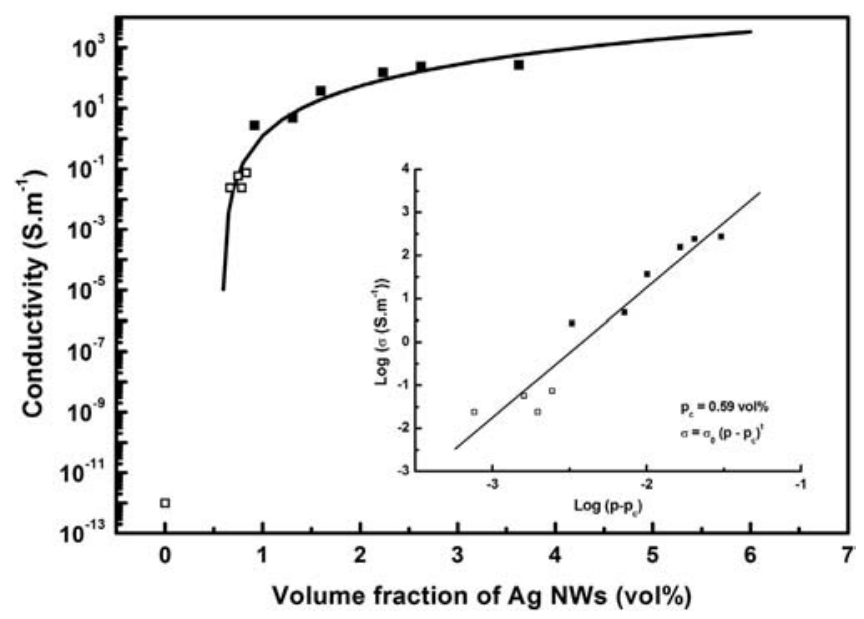

Fig. 6. Dependence of the dc conductivity $\left(\sigma_{\mathrm{dc}}\right)$ on the Ag nanowires volume fraction in PA11 matrix at $25{ }^{\circ} \mathrm{C}$. ( $\square$ ) Conductivity measurements measured by SDD and ( $\square$ ) conductivity measurements four wires.

by DSC (Fig. 3). The samples filled with the highest volume content of NWs (3.6 vol.\%) exhibit a difference with a decrease of the $G^{\prime}$ modulus and a decrease of $8{ }^{\circ} \mathrm{C}$ of the $T_{\alpha}$ (Fig. 8). The $G^{\prime}$ and $T_{\alpha}$ decrease could be explained by the presence of small aggregates with the highest content of NWs. $T_{\alpha}$ is more affected than $T_{\mathrm{g}}$ measured by DSC. The DMA sample size is larger than DSC and the process to obtain this sample probably favors aggregation phenomenon. Nanocomposites show a slight broadening of the viscoelastic response on Fig. 8. This broadening suggests that NWs increase the heterogeneity of the amorphous phase.

Fig. 9 shows the $G^{\prime}$ moduli evolution in the glassy and rubbery states. Below 2.5\% vol. NWs content, we note the same increase of the stiffening in the two states. This phenomenon is different from previous results on PA11 filled with quasi spherical $\mathrm{BaTiO}_{3}$ nanoparticles [35]. The NWs play the role of additional entanglements for polymeric chains in the amorphous phase [11] and decrease their mobility. Furthermore, the modulus reinforcement is higher in PA11. It could be explained by a stronger interaction of amide groups than fluoride with metallic nanowires.

Above $2.5 \%$ vol. NWs content, two behaviors are observed. Cristallinity ratio and interactions are responsible for these evolutions. The NWs influence on matrix crystallinity is different in PA11 and P(VDF-TrFE)[11]. In $\mathrm{P}(\mathrm{VDF}-\mathrm{TrFE})$, the crystallinity decreases drastically resulting first a large increase of amorphous phase and a difference between glassy and rubbery state behavior. The PA11 crystallinity is invariant with the NWs content. So, the amorphous phase fraction is constant. The $G^{\prime}$ modulus of glassy and vitreous state increases in the same range.

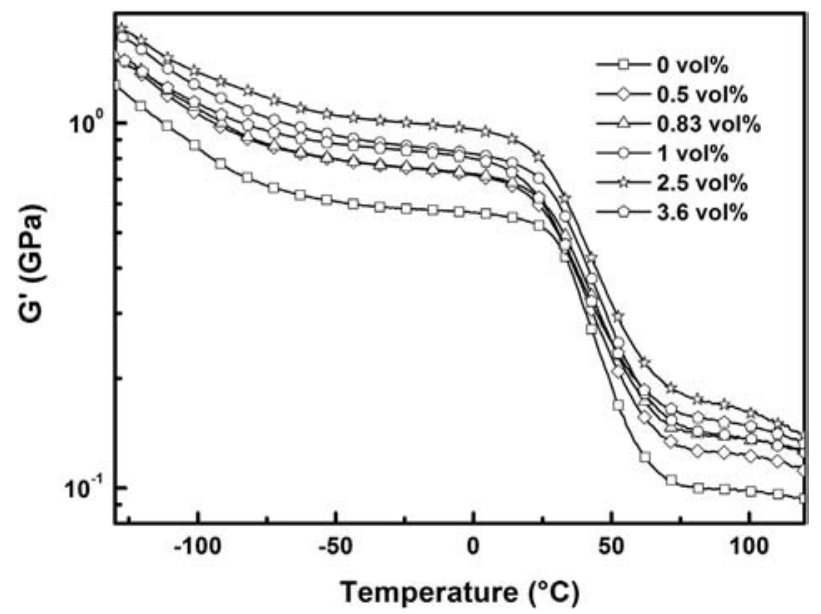

Fig. 7. Storage shear modulus $G^{\prime}$ versus temperature for PA11/Ag nanowires from 0 vol.\% to 3.6 vol.\%.

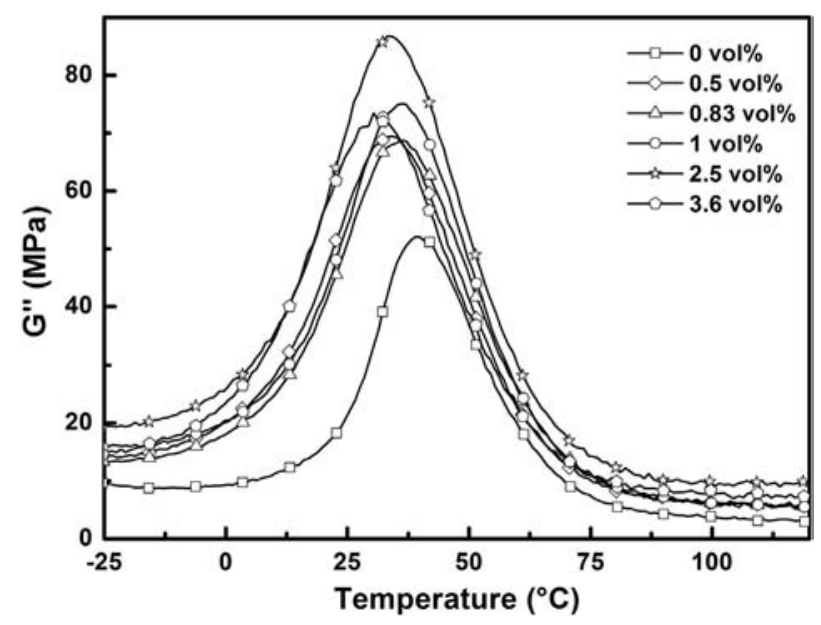

Fig. 8. Dissipative shear modulus $G^{\prime \prime}$ of the mechanical $\beta$ and $\alpha$ relaxations versus temperature for PA11/Ag nanowires from 0 vol.\% to 3.6 vol.\%.

\section{Conclusion}

Polyamide 11/Ag nanowires nanocomposites were prepared. The method used in this study has been developed to provide highly conductive thermoplastic composites while preserving the mechanical properties of pristine PA11. The percolation threshold has been observed for 0.59 vol.\% of Ag NWs. This value is the lowest measured with metallic nanoparticles filled in thermoplastic matrix and comparable with values obtained with CNTs [5]. The combination of solvent way method to introduce nanowires in PA11 and extrusion to obtain directly usable pellets for industrial application was developed. This original method provides excellent results in dispersion for low filled nanocomposites ( $<3.6 \mathrm{vol} . \%)$ and ensures the preservation of aspect ratio of NWs. The PA11 characteristics as $T_{\mathrm{g}}$ and crystallinity are unaffected by the insertion of low content of NWs. The mechanical properties have been preserved above $p_{\mathrm{c}}$ for lower filler content. The level of conductivity reached above the percolation threshold $\left(10^{2} \mathrm{~S} \mathrm{~m}^{-1}\right)$ corresponds to highly conductive composites filled with metallic particles. The dispersion of $\mathrm{Ag} \mathrm{NWs}$ in thermoplastic semi-crystalline PA11 permits to obtain low filled conductive composites in polyamide. It is important to emphasize that electrically percolated AgNW nanocomposites with $p_{\mathrm{c}}$ comparable to CNTs [5] reach an electrical conductivity $10^{3}$ times higher than the one of CNTs nanocomposites $[4,27,36]$. Despite moderate aspect ratio regarding CNTs, metallic

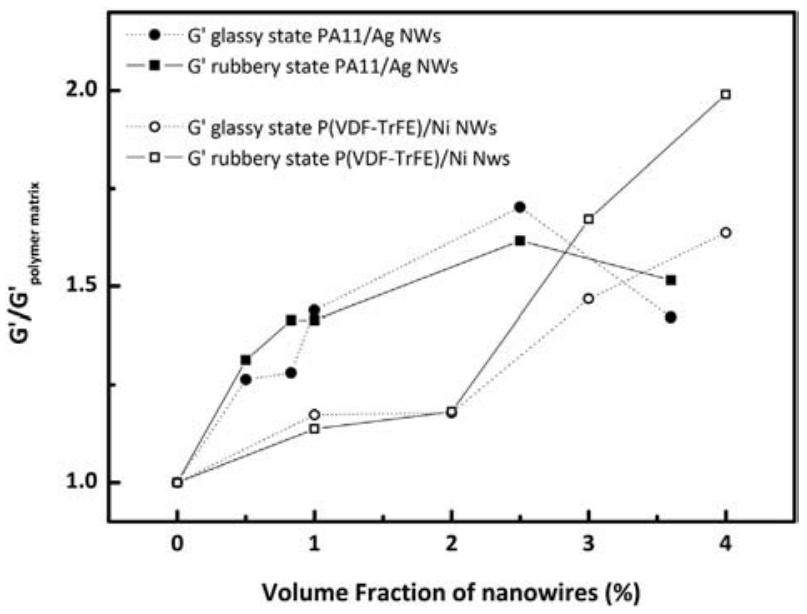

Fig. 9. Storage shear modulus $G^{\prime}$ of nanocomposites measured on glassy plateau $\left(T_{\alpha}-50{ }^{\circ} \mathrm{C}\right)$ and on rubbery plateau $\left(T_{\alpha}+50^{\circ} \mathrm{C}\right)$ versus the NWs volume fraction (vol.\%). $G^{\prime}$ is normalized to the $G^{\prime}$ of the neat polymer matrix. 
nanowires represent a promising route for optimizing the electrical conduction of polymer matrix composites.

\section{References}

[1] F. Lux, J. Mater. Sci. 28 (1993) 285-301.

[2] T.A. Ezquerra, M. Kulescza, C. Santa-Cruz, F.J. Baltá-Calleja, Adv. Mater. 2 (1990) 597-600.

[3] A.I. Medalia, Rubber Chem. Technol. 59 (1986) 432-454.

[4] S. Barrau, P. Demont, A. Peigney, C. Laurent, C. Lacabanne, Macromolecules 36 (2003) 5187-5194.

[5] D. Carponcin, E. Dantras, G. Aridon, F. Levallois, L. Cadiergues, C. Lacabanne, Compos. Sci. Technol. 72 (2012) 515-520.

[6] W. Bauhofer, S.C. Schulz, A.E. Eken, T. Skipa, D. Lellinger, I. Alig, E.J. Tozzi, D.J. Klingenberg, Polymer 51 (2010) 5024-5027.

[7] G. Morales, M.I. Barrena, J.M. Gómez de Salazara, C. Merino, D. Rodríguez, Compos. Struct. 92 (2010) 1416-1422.

[8] G.A. Gelves, U. Sundararaj, J.A. Haber, Macromol. Rapid. Comm. 26 (2005) 1677-1681.

[9] A. Lonjon, L. Laffont, P. Demont, Dantras, C. Lacabanne, J. Phys. Chem. C 113 (2009) 12002-12006.

[10] A. Lonjon, P. Demont, E. Dantras, C. Lacabanne, J. Non-Cryst. Solids 358 (2012) 3074-3078.

[11] A. Lonjon, P. Demont, E. Dantras, C. Lacabanne, J. Non-Cryst. Solids 358 (2011) 236-240.

[12] Y.G. Sun, B. Gates, B. Mayers, Y.N. Xia, Nano Lett. 2 (2002) 165-168.

[13] Y.G. Sun, Y.D. Yin, B.T. Mayers, T. Herricks, Y.N. Xia, Chem. Mater. 14 (2002) 4736-4745.

[14] J.W. Cho, D.R. Paul, Polymer 42 (2001) 1083-1094.

[15] S.C. Schulz, G. Faiella, S.T. Buschhorn, L.A.S.A. Prado, M. Giordano, K. Schulte, W. Bauhofer, Eur. Polym. J. 47 (2011) 2069-2077.

[16] S.R. Athreya, K. Kalaitzidou, S. Das, Adv. Mater. Sci. Eng. A 527 (2010) 2637-2642.

[17] Stauffer G., Taylor \& Francis: London, 1985.

[18] D.M. Bigg, Advances in Polymer Science 119 (1995) 1-30.
[19] I. Balberg, C.H. Anderson, S. Alexander, N. Wagner, Phys. Rev. B 30 (1984) 3933-3943.

[20] I. Balberg, N. Binenbaum, Phys. Rev. Lett. 52 (1984) 1465-1468

[21] C. Sender, E. Dantas, L. Dantras-Laffont, M.H. Lacoste, J. Dandurand, M. Mauzac, J.L. Lacout, C. Lavergne, A. Bernes, C. Lacabanne, J. Biomed. Mater. Res. B Appl. Biomater. 83B (2007) 628-635.

[22] E. Devaux, S. Bourbigot, A. El Achari, J. Appl. Polym. Sci. 86 (2002) 2416-2423.

[23] E. Logakis, C. Pandis, V. Peoglos, P. Pissis, C. Stergiou, J. Pionteck, P. Pötschke, M. Micusik, M. Omastova, Polym. Sci. B Polym. Phys. 47 (2009) 764-774.

[24] A. Lonjon, L. Laffont, P. Demont, Dantras, C. Lacabanne, J. Phys. D Appl. Phys. 43 (2010) 345401.

[25] B. Lin, G.A. Gelves, J.A. Haber, P. Pötschke, U. Sundararaj, Macromol. Mater. Eng. 293 (2008) 631-640.

[26] Y. Tao, Y. Xia, H. Wang, F. Gong, H. Wu, G. Tao, IEEE Trans. Adv. Packag. 32 (2009) 589-592.

[27] Y. Mamunya, Electrical Properties in Carbon Nanotubes as Conductive Filler in Segregated Polymer Composites, in: S. Yellampalli (Ed.), INTECH, 2011, pp. 173-196.

[28] Stauffer D., Aharony A., Taylor \& Francis: London, 1985.

[29] W. Bauhofer, J.Z. Kovacs, Compos. Sci. Technol. 69 (2009) 1486-1498.

[30] M. Karttunen, P. Ruuskanen, V. Pitkanen, W.M. Albers, J. Elec. Materi. 37 (2008) 951-954.

[31] M.P. Alvarez, V.H. Poblete, M.E. Pilleux, V.M. Fuenzalida, J. Appl. Polym. Sci. 99 (2006) 3005-3008.

[32] H.P. Xu, Z.M. Dang, Chem. Phys. Lett. 438 (2007) 196-202.

[33] V. Augelli, T. Ligonzo, M.C. Masellis, M.F. Muscarella, L. Schiavulli, A. Valentini, J. Appl. Phys. 90 (2001) 1362-1367.

[34] S. Huang, M. Wang, T.X. Liu, W.D. Zhang, W.C. Tjiu, C.B. He, X.H. Lu, Polym. Eng Sci. 49 (2009) 1063-1068.

[35] J.F. Capsal, C. Pousserot, E. Dantras, J. Dandurand, C. Lacabanne, Polymer 51 (2010) 5207-5211.

[36] A. Lonjon, S. Barrau, E. Dantras, P. Demont, C. Lacabanne, Eur. J. Electr. Eng. 12 (2009) 423-431. 